\title{
Supporting Information \\ Hollow Biphase Cobalt Nickel Perselenide Spheres Derived from Metal Glycerol Alkoxides for High-performance Hybrid Supercapacitors
}

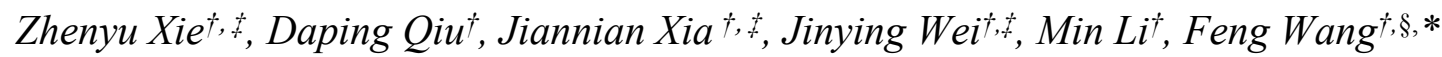
Ru Yang ${ }^{\dagger}, \not \dot{*}, *$

† State Key Laboratory of Chemical Resource Engineering, Beijing Key Laboratory of Electrochemical Process and Technology for Materials, Beijing University of Chemical Technology, Beijing 100029, P. R. China.

$\$$ Changzhou Institute of Advanced Materials, Beijing University of Chemical Technology, Changzhou, Jiangsu 213000, P. R.China.

$\S$ Beijing Advanced Innovation Center for Soft Matter Science and Engineering, Beijing University of Chemical Technology, Beijing 100029, P. R.China.

* Corresponding authors:

wangf@mail.buct.edu.cn (Feng Wang);

ruyang@mail.buct.edu.cn (Ru Yang).

\section{Materials}

$\mathrm{Ni}\left(\mathrm{NO}_{3}\right)_{2} \cdot 6 \mathrm{H}_{2} \mathrm{O}, \mathrm{Co}\left(\mathrm{NO}_{3}\right)_{2} \cdot 6 \mathrm{H}_{2} \mathrm{O}, \mathrm{SeO}_{2}$, pyrrole (Py), aluminium isopropoxide and ammonium persulfate were purchased from Aladdin Industrial Corporation. Isopropanol, glycerol and ethanol were purchased from Beijing Tongguang Fine Chemical Company. Phytic acid (PA) were purchased from Tianjin Guangfu Fine 
Chemical Company. All reagents were used without further purification.

\section{Synthesis of $\mathrm{N}_{2} \mathrm{C}_{4}$-glycerol and $\mathrm{N}_{2} \mathrm{C}_{4}$-isopropanol}

In order to clearly understand the composition of the precursors, the comparative samples $\left(\mathrm{N}_{2} \mathrm{C}_{4}\right.$-glycerol and $\mathrm{N}_{2} \mathrm{C}_{4}$-isopropanol) were synthesized in pure glycerol and in pure isopropanol, respectively. The synthesis process is as follows: $\mathrm{Ni}\left(\mathrm{NO}_{3}\right)_{2} \cdot 6 \mathrm{H}_{2} \mathrm{O}$ and $\mathrm{Co}\left(\mathrm{NO}_{3}\right)_{2} \cdot 6 \mathrm{H}_{2} \mathrm{O}$ with molar ratios of $2 / 4$, in which the molar sum of $\mathrm{Co}$ atom and

$\mathrm{Ni}$ atom is $3 \mathrm{mmol}$, were dissolved into $160 \mathrm{ml}$ of glycerol or isopropanol by strongly stirring to form a transparent solution. The clear solution was poured into a $200 \mathrm{ml}$ Teflon-lined stainless steel autoclave and maintained at $180{ }^{\circ} \mathrm{C}$ for $6 \mathrm{~h}$ in an oven. After cooling to room temperature, the precursors, $\mathrm{N}_{2} \mathrm{C}_{4}$-glycerol and $\mathrm{N}_{2} \mathrm{C}_{4}$-isopropanol, were filtrated and washed several times with ethanol, finally dried at $70{ }^{\circ} \mathrm{C}$.

\section{Synthesis of Hierarchical Porous Carbon}

The equal volumes of Py and PA were first dissolved into $5 \mathrm{ml}$ deionized water, and dispersed evenly by ultrasonic for $10 \mathrm{~min}$. Then an ammonium persulfate aqueous solution was quickly added into above solution with vigorous stirring and a large amount of black products could be observed after half a minute. After polymerization for $6 \mathrm{~h}$, the as-precursors were filtrated and washed with deionized water to remove the ammonium persulfate, then dried at $70{ }^{\circ} \mathrm{C}$. The precursors were finely ground and put into a tube furnace for pyrolysis at $400{ }^{\circ} \mathrm{C}$ for $2 \mathrm{~h}$ under nitrogen atmosphere. The pyrolysis products and $\mathrm{KOH}$ were mixed evenly at a mass ratio of $1 / 4$, and then activated at $900{ }^{\circ} \mathrm{C}$ for $2 \mathrm{~h}$ under argon atmosphere. After cooling to room 
temperature, the samples were washed with dilute hydrochloric acid and deionized water for several times, and finally dried at $80^{\circ} \mathrm{C}$.

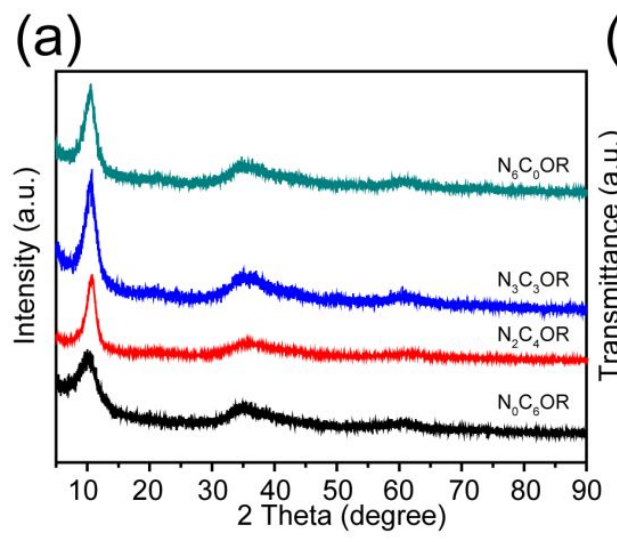

(b)

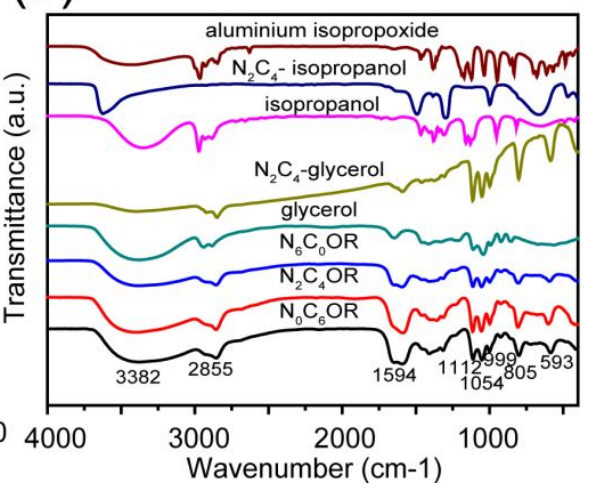

Figure $\mathrm{S} 1$ (a) XRD patterns of $\mathrm{N}_{\mathrm{x}} \mathrm{C}_{\mathrm{y}} \mathrm{OR}$ and (b) FTIR spectra of $\mathrm{N}_{\mathrm{x}} \mathrm{C}_{\mathrm{y}} \mathrm{OR}$, glycerol, $\mathrm{N}_{2} \mathrm{C}_{4}$-glycerol, $\mathrm{N}_{2} \mathrm{C}_{4}$-isopropanol, glycerol, isopropanol and aluminium isopropoxide.

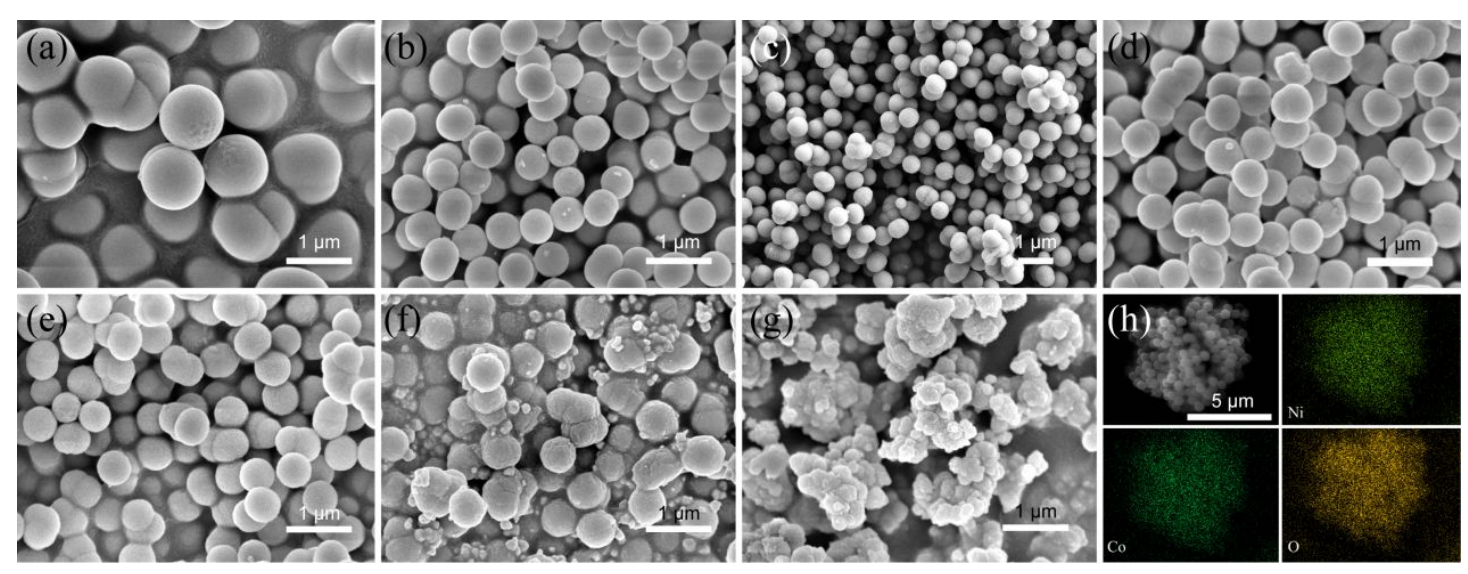

Figure S2 SEM images of the precursors of $\mathrm{Ni} / \mathrm{Co}$ alkoxide with different $\mathrm{Ni} / \mathrm{Co}$ ratios, (a) $\mathrm{N}_{0} \mathrm{C}_{6} \mathrm{OR}$, (b) $\mathrm{N}_{1} \mathrm{C}_{5} \mathrm{OR}$, (c) $\mathrm{N}_{2} \mathrm{C}_{4} \mathrm{OR}$ (d) $\mathrm{N}_{3} \mathrm{C}_{3} \mathrm{OR}$, (e) $\mathrm{N}_{4} \mathrm{C}_{2} \mathrm{OR}$, (f) $\mathrm{N}_{5} \mathrm{C}_{1} \mathrm{OR}$, (g) $\mathrm{N}_{6} \mathrm{C}_{0} \mathrm{OR}$, (h) mapping picture of $\mathrm{N}_{2} \mathrm{C}_{4} \mathrm{OR}$. 


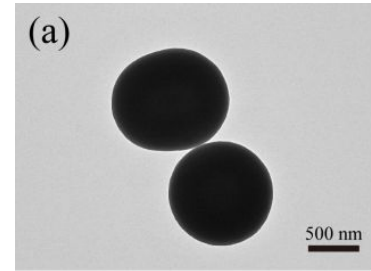

(e)

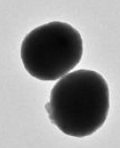

(b)

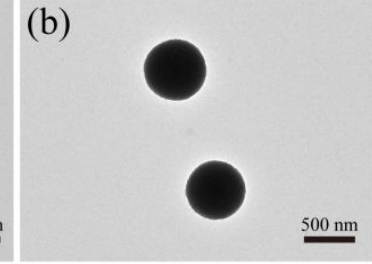

(f)

$\underline{500 \mathrm{~nm}}$

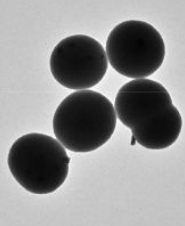

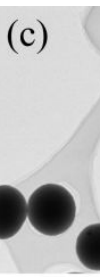

(g)
$500 \mathrm{~nm}$

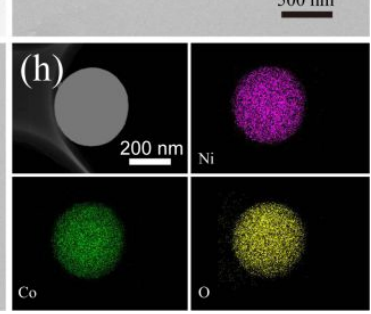

Figure S3 TEM of the precursors of Ni/Co alkoxide with different Ni/Co ratio, (a)

$\mathrm{N}_{0} \mathrm{C}_{6} \mathrm{OR}$, (b) $\mathrm{N}_{1} \mathrm{C}_{5} \mathrm{OR}$, (c) $\mathrm{N}_{2} \mathrm{C}_{4} \mathrm{OR}$, (d) $\mathrm{N}_{3} \mathrm{C}_{3} \mathrm{OR}$, (e) $\mathrm{N}_{4} \mathrm{C}_{2} \mathrm{OR}$, (f) $\mathrm{N}_{5} \mathrm{C}_{1} \mathrm{OR}$, (g) $\mathrm{N}_{6} \mathrm{C}_{0} \mathrm{OR}$, (h) mapping picture of $\mathrm{N}_{2} \mathrm{C}_{4} \mathrm{OR}$.

(a)

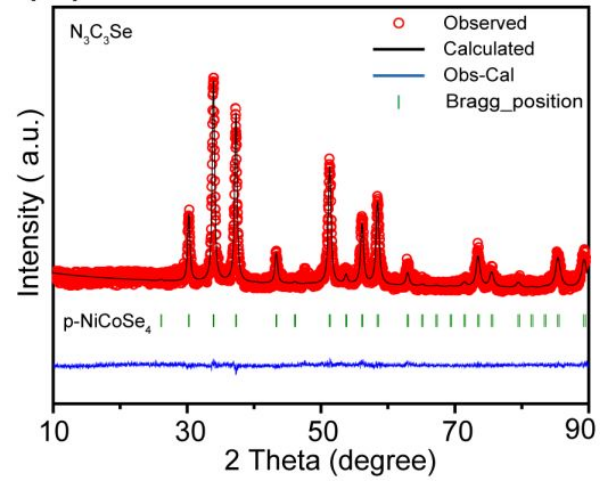

(b)

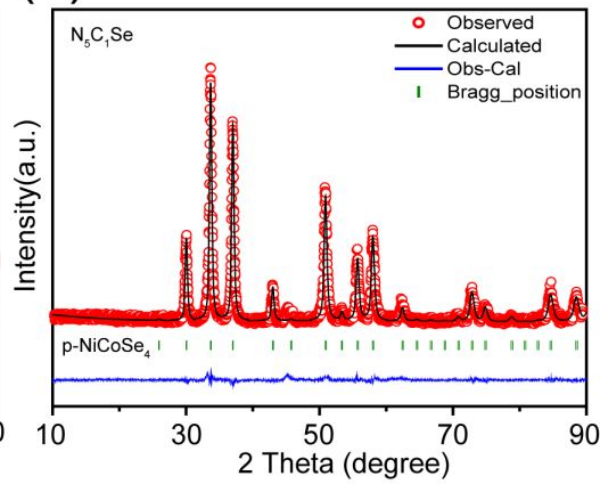

Figure S4 Rietveld-refined XRD (fullprof) patterns of (a) $\mathrm{N}_{3} \mathrm{C}_{3} \mathrm{Se}$ and (b) $\mathrm{N}_{5} \mathrm{C}_{1} \mathrm{Se}$.

(a)

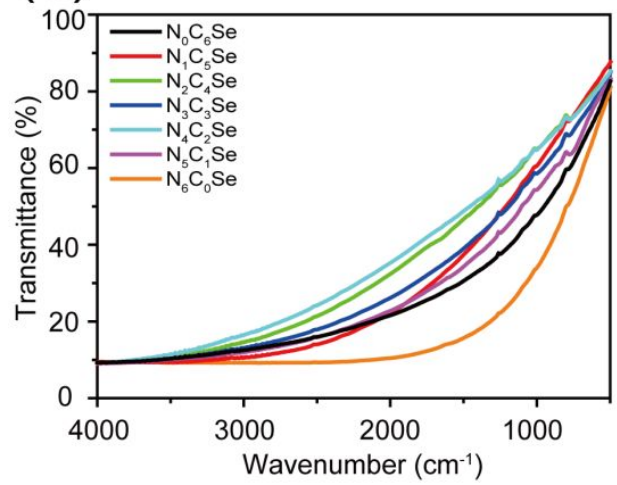

(b)

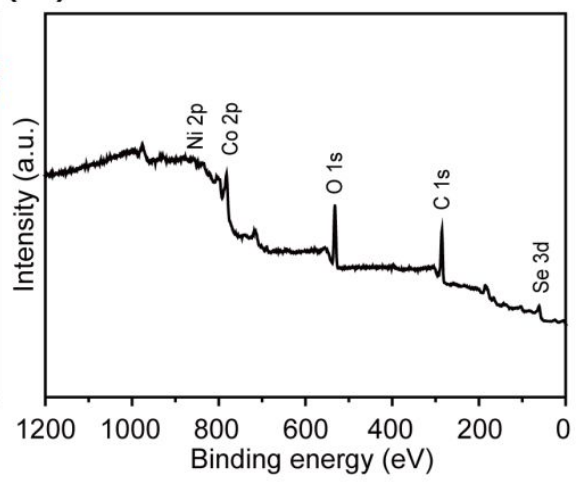

Figure S5 (a) FTIR of $\mathrm{N}_{\mathrm{x}} \mathrm{C}_{\mathrm{y}} \mathrm{Se}$, (b) Full XPS spectra of $\mathrm{N}_{2} \mathrm{C}_{4} \mathrm{Se}$. 
(a)

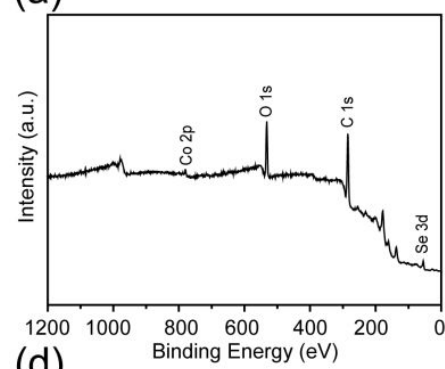

(d)

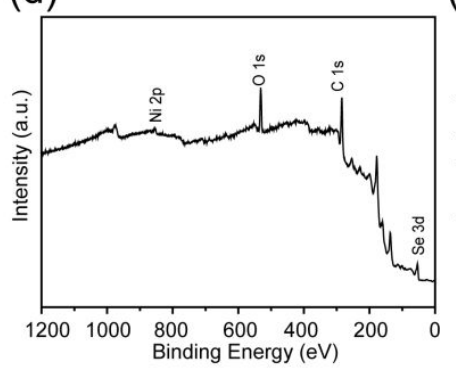

(b)

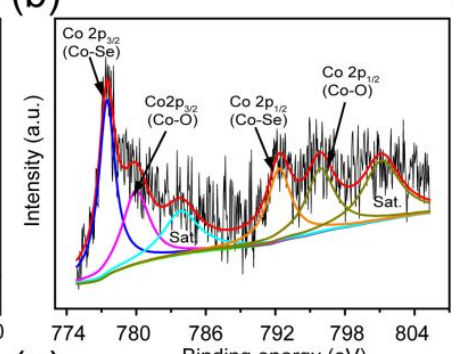

(e)

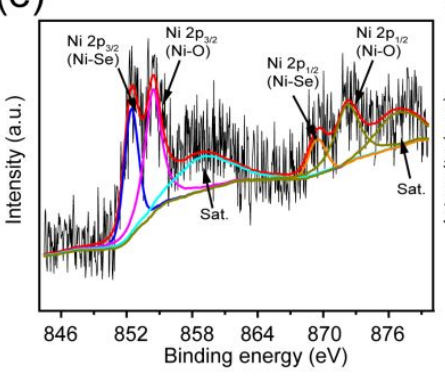

(c)

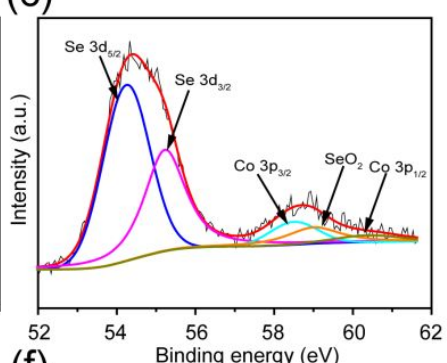

(f)

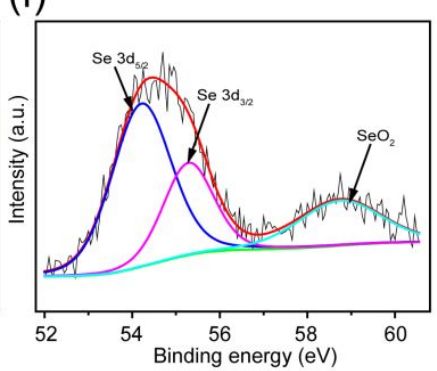

Figure S6 Full XPS spectra of (a) $\mathrm{N}_{0} \mathrm{C}_{6} \mathrm{Se}$ and (d) $\mathrm{N}_{6} \mathrm{C}_{0} \mathrm{Se}$. High-resolution XPS spectra: (b) Co $2 \mathrm{p}$ of $\mathrm{N}_{0} \mathrm{C}_{6} \mathrm{Se}$, (e) Ni $2 \mathrm{p}$ of $\mathrm{N}_{6} \mathrm{C}_{0} \mathrm{Se}$, Se $3 \mathrm{~d}$ of (c) $\mathrm{N}_{0} \mathrm{C}_{6} \mathrm{Se}$ and (f) $\mathrm{N}_{6} \mathrm{C}_{0} \mathrm{Se}$.

(a)

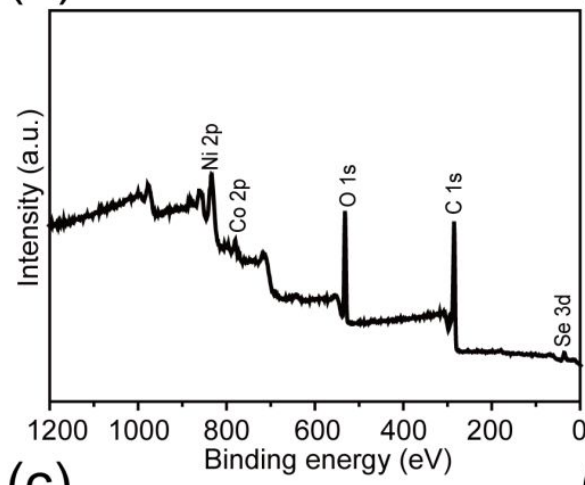

(c)

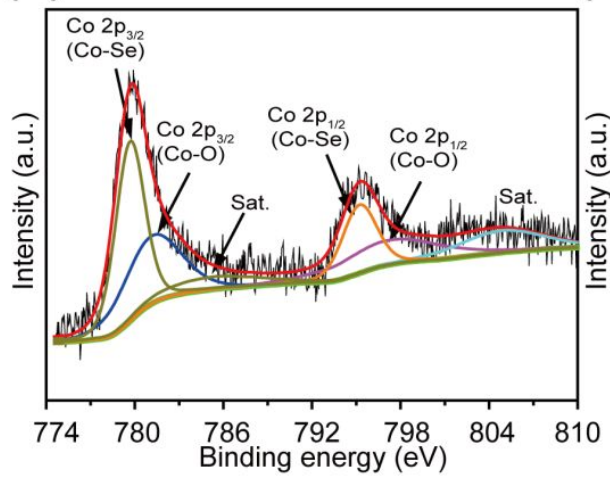

(b)

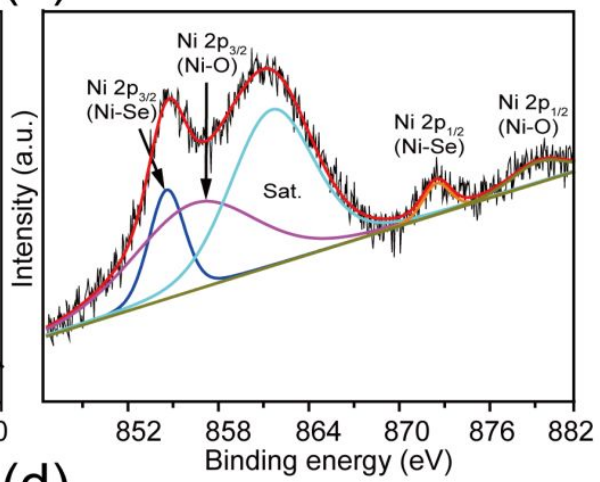

(d)

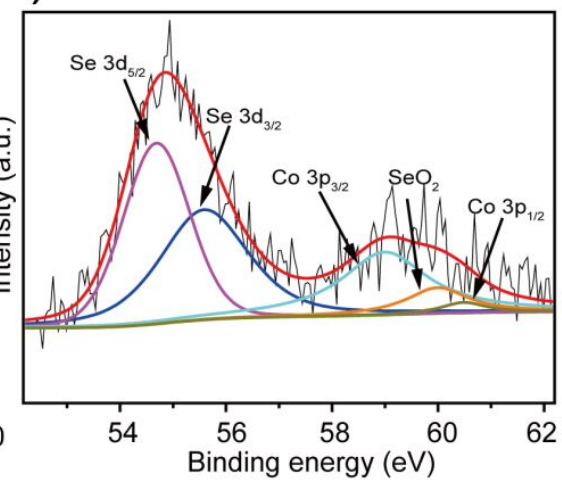

Figure S7 XPS spectra of $\mathrm{N}_{3} \mathrm{C}_{3} \mathrm{Se}$, (a) full spectrum, high-resolution spectra for (b) $\mathrm{Ni}$ 
$2 \mathrm{p}$, (c) Co $2 \mathrm{p}$ and (d) Se $3 \mathrm{~d}$.

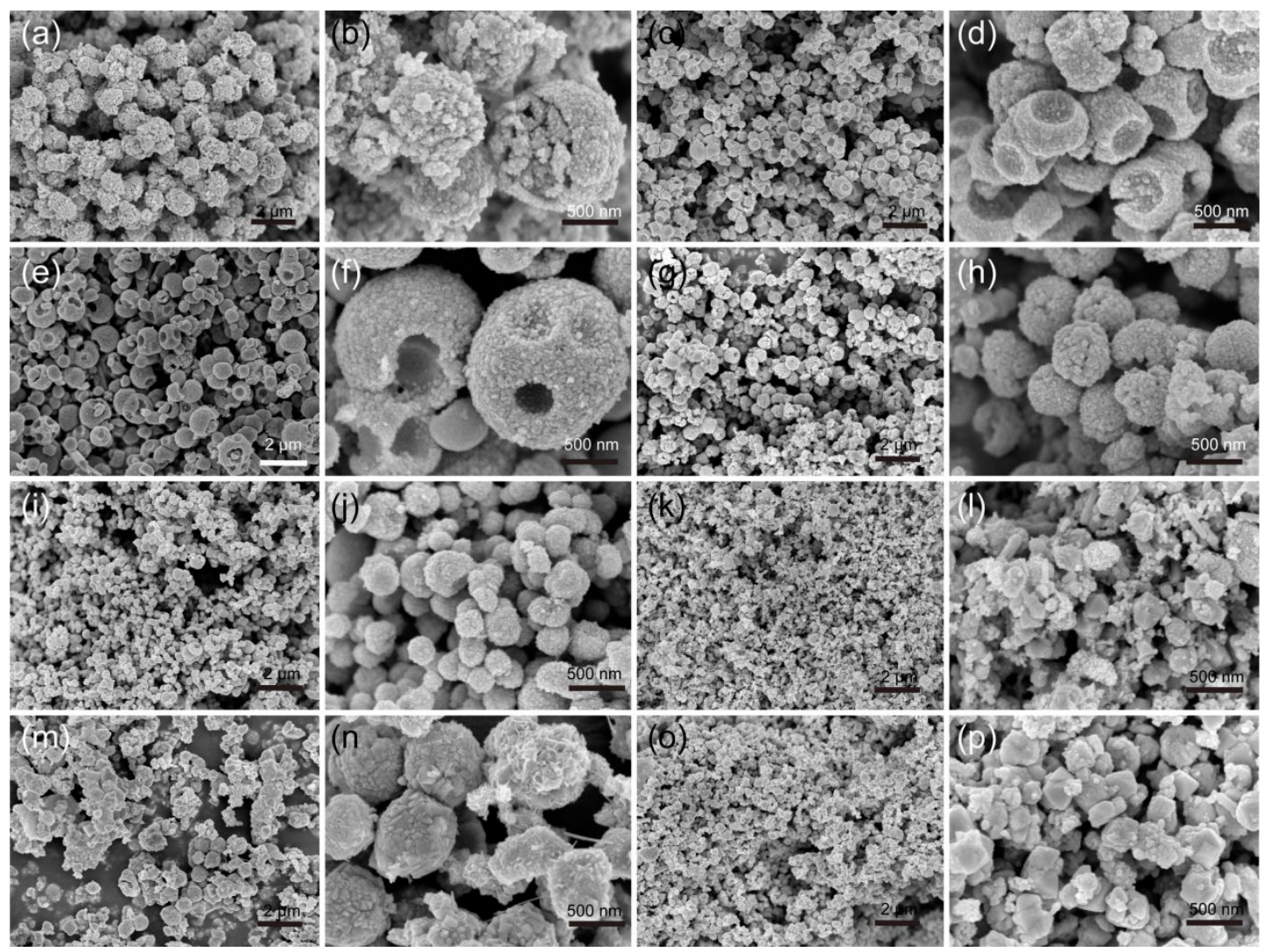

Figure S8 SEM images of samples prepared at different Ni/Co ratios: (a), (b) $\mathrm{N}_{0} \mathrm{C}_{6} \mathrm{Se}$;

(c), (d) $\mathrm{N}_{1} \mathrm{C}_{5} \mathrm{Se}$; (e), (f) $\mathrm{N}_{1.5} \mathrm{C}_{4.5} \mathrm{Se}$; (g), (h) $\mathrm{N}_{2} \mathrm{C}_{4} \mathrm{Se}$; (i), (j) $\mathrm{N}_{3} \mathrm{C}_{3} \mathrm{Se}$; (k), (l) $\mathrm{N}_{4} \mathrm{C}_{2} \mathrm{Se}$;

(m), (n) $\mathrm{N}_{5} \mathrm{C}_{1} \mathrm{Se}$ and (o), (p) $\mathrm{N}_{0} \mathrm{C}_{6} \mathrm{Se}$.

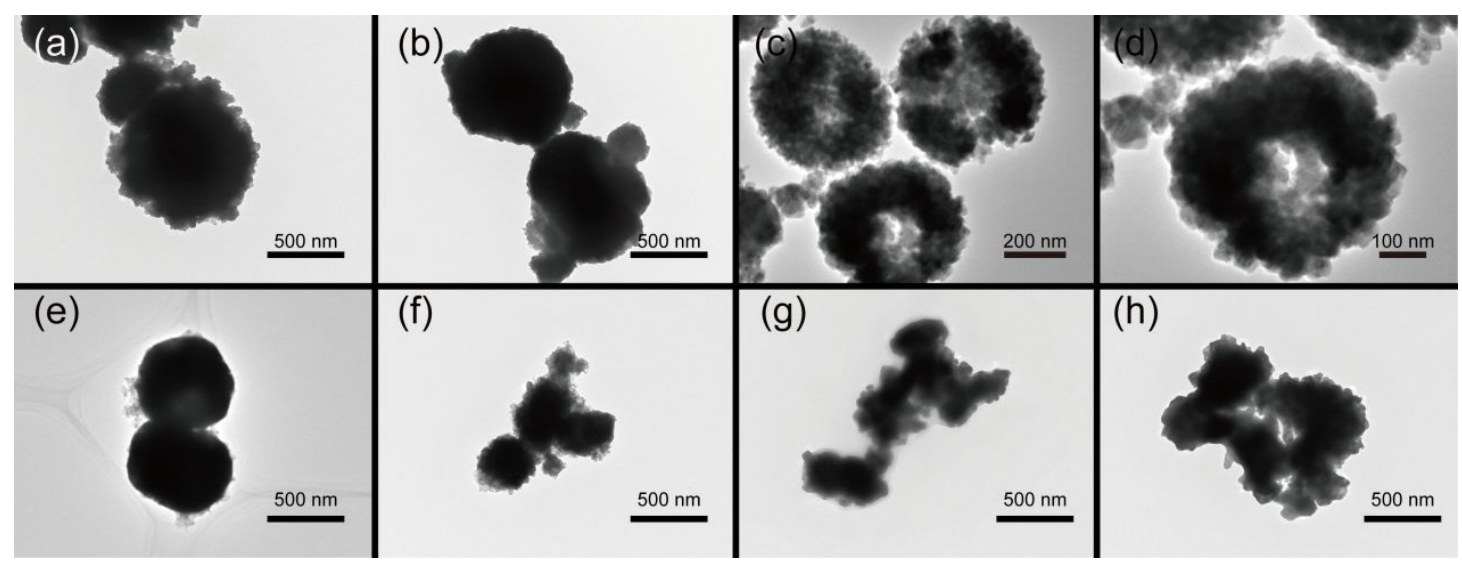

Figure S9 TEM images of samples prepared at different Ni/Co ratios: (a) $\mathrm{N}_{0} \mathrm{C}_{6} \mathrm{Se}$, (b)

$\mathrm{N}_{1} \mathrm{C}_{5} \mathrm{Se}$, (c) (d) $\mathrm{N}_{2} \mathrm{C}_{4} \mathrm{Se}$, (e) $\mathrm{N}_{3} \mathrm{C}_{3} \mathrm{Se}$, (f) $\mathrm{N}_{4} \mathrm{C}_{2} \mathrm{Se},(\mathrm{g}) \mathrm{N}_{5} \mathrm{C}_{1} \mathrm{Se}$ and (h) $\mathrm{N}_{6} \mathrm{C}_{0} \mathrm{Se}$. 


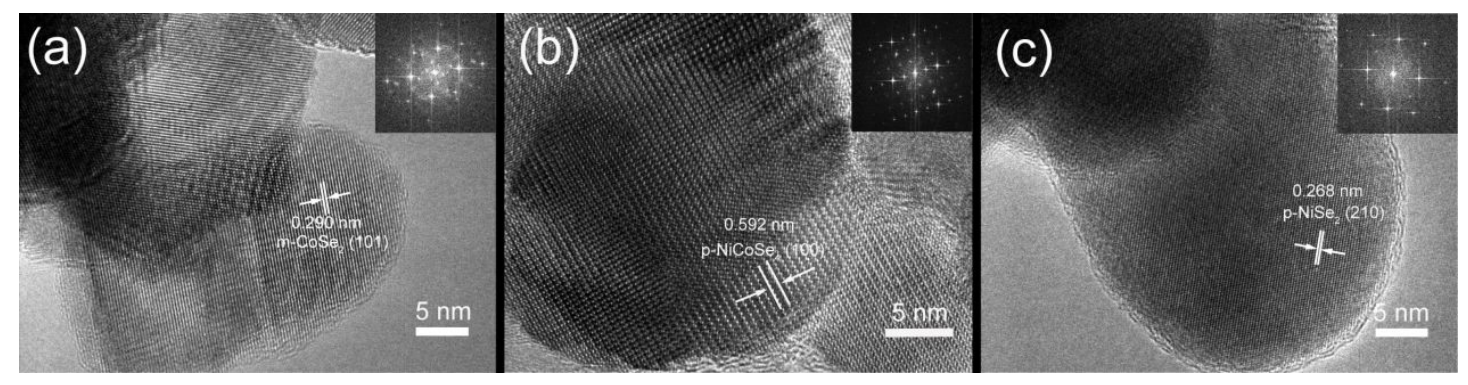

Figure S10 HRTEM images (a) $\mathrm{N}_{0} \mathrm{C}_{6} \mathrm{Se}$, (b) $\mathrm{N}_{3} \mathrm{C}_{3} \mathrm{Se}$, (c) $\mathrm{N}_{6} \mathrm{C}_{0} \mathrm{Se}$.
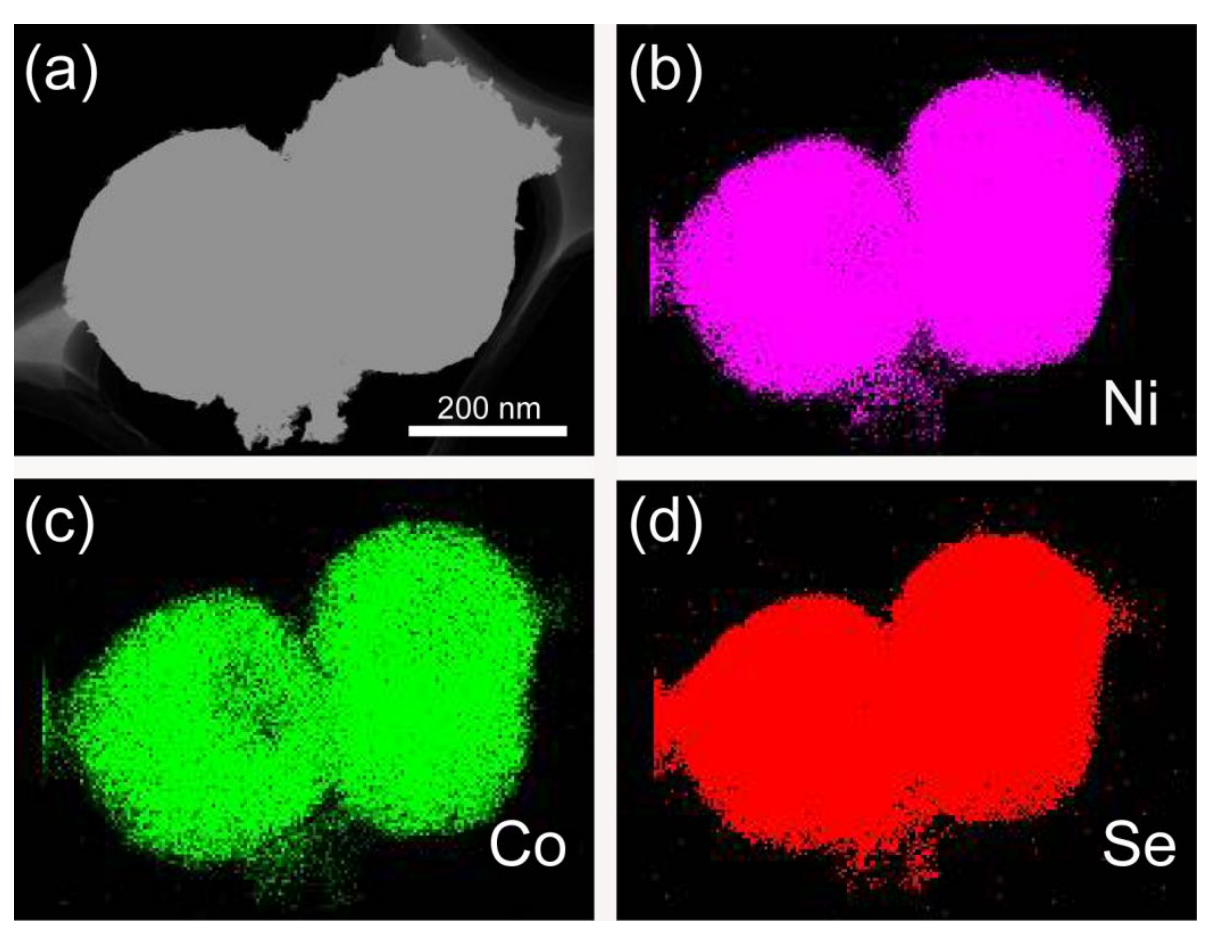

Figure $\mathrm{S} 11$ Mapping pictures of $\mathrm{N}_{3} \mathrm{C}_{3} \mathrm{Se}$.
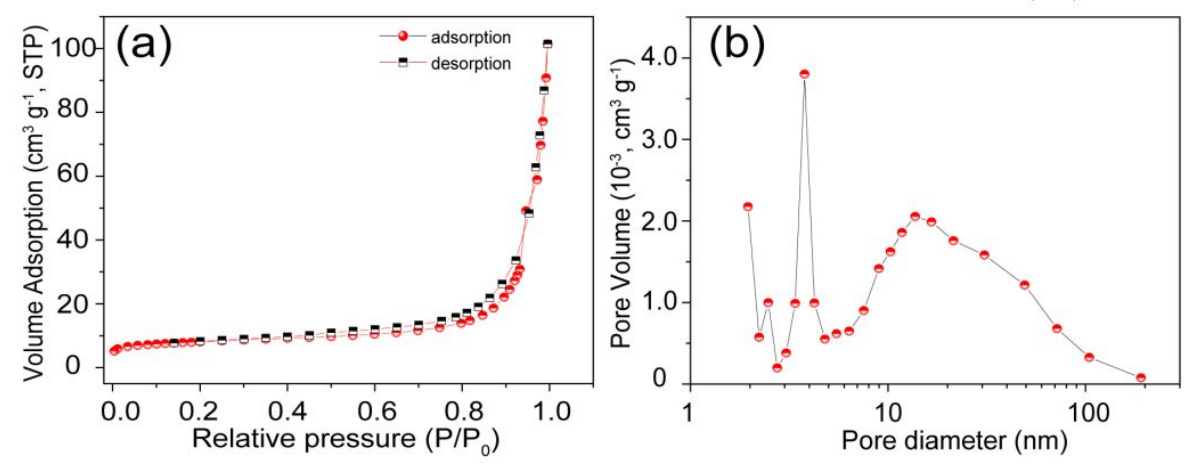

Figure $\mathrm{S} 12 \mathrm{~N}_{2}$ adsorption-desorption isotherm and pore size distribution of $\mathrm{N}_{2} \mathrm{C}_{4} \mathrm{Se}$. 

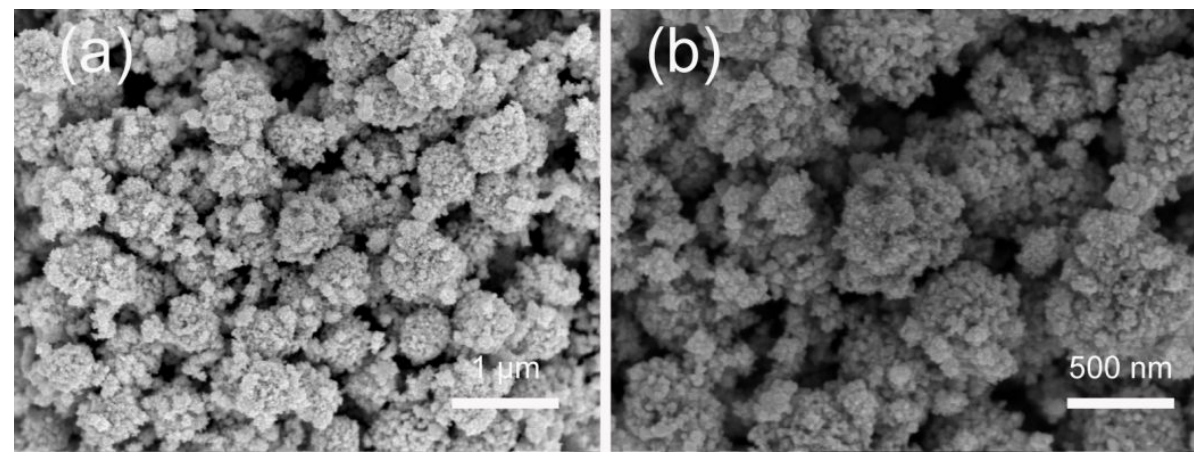

Figure $\mathrm{S} 13$ (a), (b) SEM images of $\mathrm{N}_{2} \mathrm{C}_{4}$ Se after 5000 cycles.
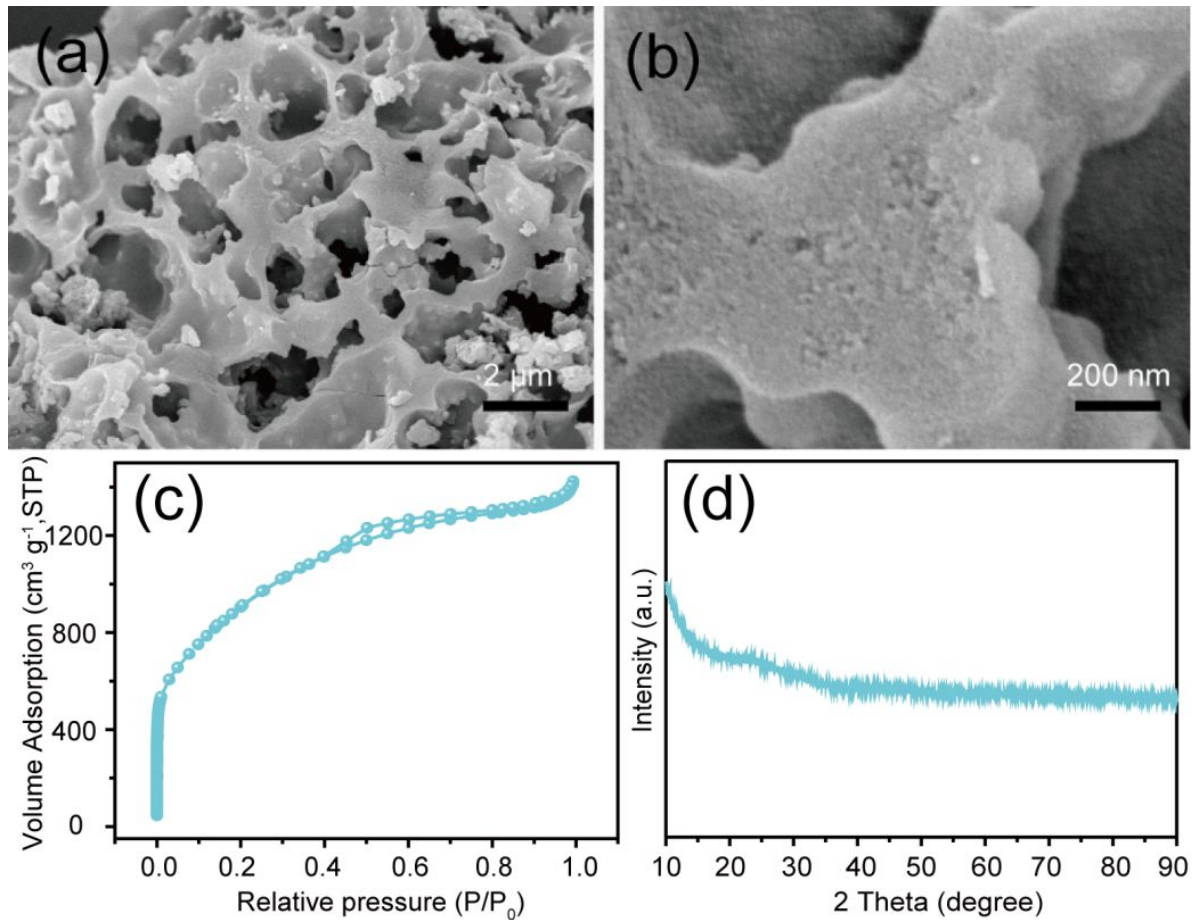

Figure S14 (a), (b) SEM images, (c) $\mathrm{N}_{2}$ adsorption-desorption isotherm and (d) XRD patterns of HPC. 
(a)

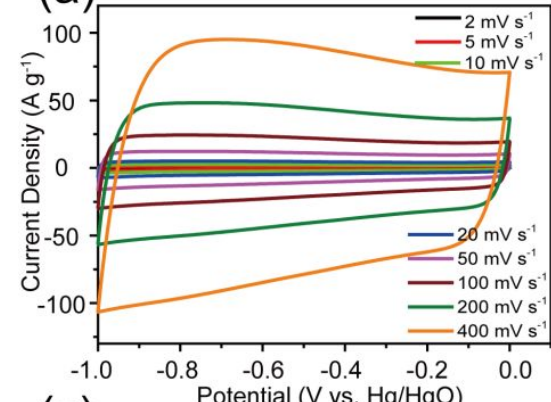

(c)

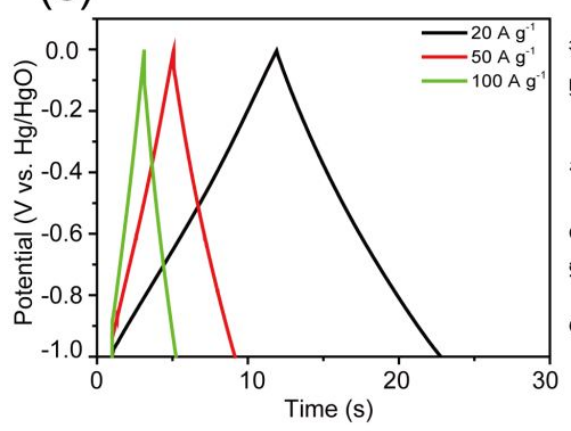

(b)

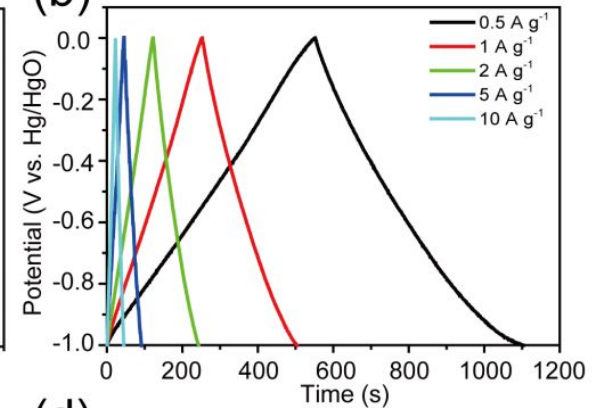

(d)

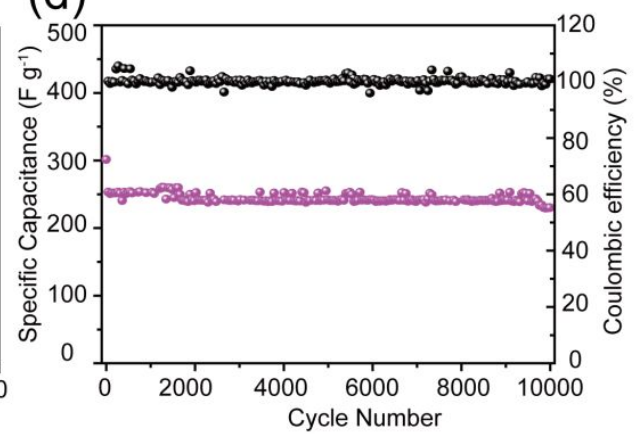

Figure S15 Electrochemical performance of the HPC electrode. (a) CV curves of the HPC electrode at various scan rates from $2 \mathrm{mV} / \mathrm{s}$ to $100 \mathrm{mV} / \mathrm{s}$, (b) (c) GCD curves of the HPC electrode at different current densities from $2 \mathrm{~A} / \mathrm{g}$ to $100 \mathrm{~A} / \mathrm{g}$, (d) Long cycles of the HPC electrode.

Table S1 Elemental compositions of the samples obtained by ICP

\begin{tabular}{lcc}
\hline Samples & Ni/Co feed molar ratio of raw materials & Ni/Co molar ratio in $\mathrm{N}_{\mathrm{x}} \mathrm{C}_{\mathrm{y}}$ Se by ICP \\
\hline $\mathrm{N}_{1} \mathrm{C}_{5} \mathrm{Se}$ & 0.2 & 0.22 \\
$\mathrm{~N}_{2} \mathrm{C}_{4} \mathrm{Se}$ & 0.5 & 0.42 \\
$\mathrm{~N}_{3} \mathrm{C}_{3} \mathrm{Se}$ & 1 & 0.89 \\
$\mathrm{~N}_{4} \mathrm{C}_{2} \mathrm{Se}$ & 2 & 2.14 \\
$\mathrm{~N}_{5} \mathrm{C}_{1} \mathrm{Se}$ & 5 & 4.90 \\
\hline
\end{tabular}

Table S2 Cell parameters of as-abtained samples $\mathrm{N}_{\mathrm{x}} \mathrm{C}_{\mathrm{y}} \mathrm{Se}$

\begin{tabular}{|c|c|c|c|c|c|}
\hline \multirow{2}{*}{ Samples } & \multicolumn{3}{|c|}{ Lattice parameter } & \multirow{2}{*}{ Percentage content $(\%)$} & \multirow{2}{*}{ Composition } \\
\hline & $\mathrm{a}(\AA)$ & $\mathrm{b}(\AA)$ & c $(\AA)$ & & \\
\hline \multirow{2}{*}{$\mathrm{N}_{0} \mathrm{C}_{6} \mathrm{Se}$} & 4.864 & 5.859 & 3.636 & 96.87 & $\mathrm{~m}-\mathrm{CoSe}_{2}$ \\
\hline & 5.858 & 5.858 & 5.858 & 3.13 & $\mathrm{p}-\mathrm{CoSe}_{2}$ \\
\hline $\mathrm{N}_{1} \mathrm{C}_{5} \mathrm{Se}$ & 4.852 & 5.853 & 3.634 & 54.54 & $\mathrm{~m}-\mathrm{CoSe}_{2}$ \\
\hline
\end{tabular}




\begin{tabular}{cccccc}
\hline & 5.871 & 5.871 & 5.871 & 45.46 & p-NiCoSe \\
\hline \multirow{2}{*}{$\mathrm{N}_{2} \mathrm{C}_{4} \mathrm{Se}$} & 4.881 & 5.905 & 3.656 & 20.86 & $\mathrm{~m}-\mathrm{CoSe}_{2}$ \\
& 5.900 & 5.900 & 5.900 & 79.14 & $\mathrm{p}-\mathrm{NiCoSe}_{4}$ \\
\hline $\mathrm{N}_{3} \mathrm{C}_{3} \mathrm{Se}$ & 5.907 & 5.907 & 5.907 & 100 & $\mathrm{p}-\mathrm{NiCoSe}_{4}$ \\
\hline $\mathrm{N}_{5} \mathrm{C}_{1} \mathrm{Se}$ & 5.949 & 5.949 & 5.949 & 100 & $\mathrm{p}-\mathrm{NiCoSe}_{4}$ \\
\hline${ }^{\mathrm{a}} \mathrm{NiSe}_{2}$ & 5.963 & 5.963 & 5.963 & - & $\mathrm{p}-\mathrm{NiSe}_{2}$ \\
\hline
\end{tabular}

a: The unit cell parameters of cubic pyrite phase $\mathrm{NiSe}_{2}$ (PDF\#88-1711).

Table S3 modelized EIS spectra for different electrode materials

\begin{tabular}{lccccccc}
\hline Samples & $\mathrm{Rs}(\Omega)$ & $\mathrm{CPE}-\mathrm{T}\left(\mathrm{s}^{-\mathrm{n}}\right)$ & $\mathrm{CPE}-\mathrm{P}(\mathrm{n})$ & $\mathrm{Rp}(\Omega)$ & $\mathrm{W}_{1}-\mathrm{R}(\Omega)$ & $\mathrm{W}_{1}-\mathrm{T}(\mathrm{s})$ & $\mathrm{W}_{1}-\mathrm{P}$ \\
\hline $\mathrm{N}_{0} \mathrm{C}_{6} \mathrm{Se}$ & 0.2971 & 0.0069 & 0.8258 & 0.5042 & 47.46 & 0.9137 & 0.5 \\
$\mathrm{~N}_{1} \mathrm{C}_{5} \mathrm{Se}$ & 0.2285 & 0.0063 & 0.9093 & 0.2989 & 36.22 & 0.5269 & 0.5 \\
$\mathrm{~N}_{2} \mathrm{C}_{4} \mathrm{Se}$ & 0.2245 & 0.0027 & 0.7977 & 0.2175 & 25.82 & 0.3119 & 0.5 \\
$\mathrm{~N}_{3} \mathrm{C}_{3} \mathrm{Se}$ & 0.3995 & 0.0019 & 0.9667 & 0.1419 & 37.29 & 0.3361 & 0.5 \\
$\mathrm{~N}_{4} \mathrm{C}_{2} \mathrm{Se}$ & 0.2825 & 0.0048 & 0.9977 & 0.4846 & 40.64 & 0.6469 & 0.5 \\
$\mathrm{~N}_{5} \mathrm{C}_{1} \mathrm{Se}$ & 0.3518 & 0.0033 & 0.6869 & 4.801 & 55.97 & 3.185 & 0.5 \\
$\mathrm{~N}_{6} \mathrm{C}_{0} \mathrm{Se}$ & 0.4186 & 0.0092 & 0.8053 & 26.5 & 89.54 & 28.27 & 0.5 \\
\hline
\end{tabular}

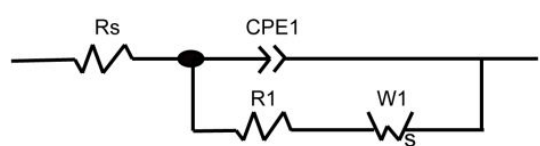

Rs: Resistance series

Rp: Resistance polarization

CPE: Constant phase element parameters

CPE-T: Double layered capacitance

CPE-P: Dispersion index

W: Warburg

$\mathrm{Z}=\mathrm{R} \times \tan (\mathrm{h})^{\wedge} \mathrm{P} /(\mathrm{I} \times \mathrm{T} \times \mathrm{W})^{\wedge} \mathrm{P}$

Ws-P: Warburg exponent phase exponent

Ws-R: Warburg resistance

Ws-T: diffusion time constant, $\mathrm{T}=\mathrm{L}^{2} / \mathrm{D}$ (L, thickness of diffusion; $\mathrm{D}$, diffusion coefficient) 
Table S4 Comparison of the electrochemical performance of $\mathrm{N}_{2} \mathrm{C}_{4} \mathrm{Se}$ and reported metal selenides in the three-electrode cells

\begin{tabular}{|c|c|c|c|c|c|}
\hline Electrode materials & Morphology & $\begin{array}{l}\text { Current } \\
\text { density }\end{array}$ & $\begin{array}{c}\text { Specific } \\
\text { capacitance }\end{array}$ & $\begin{array}{c}\text { Long cycle, } \\
\text { Retention } \\
(\%)\end{array}$ & Ref. \\
\hline $\mathrm{NiCoSe}_{2}$ & Hollow sub-microspheres & $3 \mathrm{~A} \mathrm{~g}^{-1}$ & $750 \mathrm{~F} \mathrm{~g}^{-1}$ & $5000,83.7 \%$ & 1 \\
\hline $\mathrm{NiSe}_{2}$ & $\begin{array}{l}\text { Ultrathin porous } \\
\text { nanosheeets }\end{array}$ & $1 \mathrm{~A} \mathrm{~g}^{-1}$ & $466 \mathrm{~F} \mathrm{~g}^{-1}$ & $1000,82.3 \%$ & 2 \\
\hline $\mathrm{CoSe}_{2} / \mathrm{Ni}_{0.85} \mathrm{Se}$ & Core-branched nanotube & $15 \mathrm{~mA} \mathrm{~cm}^{-2}$ & $\begin{array}{c}662 \mathrm{~F} \mathrm{~g}^{-1}(331 \mathrm{C} \\
\left.\mathrm{g}^{-1}\right)\end{array}$ & $6000,95.3 \%$ & 3 \\
\hline $\mathrm{CoSe}_{2}$ & Hollow core-branch & $1 \mathrm{~mA} \mathrm{~cm}^{-2}$ & $759.5 \mathrm{~F} \mathrm{~g}^{-1}$ & $5000,94.5 \%$ & 4 \\
\hline $\mathrm{Ni}_{0.5} \mathrm{Co}_{0.5} \mathrm{Se}_{2}$ & $\begin{array}{c}\text { Hollow sea-urchin-like } \\
\text { structure }\end{array}$ & $1 \mathrm{~A} \mathrm{~g}^{-1}$ & $\begin{array}{c}1007 \mathrm{~F} \mathrm{~g}^{-1}(524 \\
\left.\mathrm{C} \mathrm{g}^{-1}\right)\end{array}$ & $3500,91 \%$ & 5 \\
\hline$\left(\mathrm{Ni}_{0.33} \mathrm{Co}_{0.67}\right) \mathrm{Se}_{2} \mathrm{CHSs}$ & Hollow spheres & $1 \mathrm{~A} \mathrm{~g}^{-1}$ & $827.9 \mathrm{~F} \mathrm{~g}^{-1}$ & $2000,--$ & 6 \\
\hline $\mathrm{NiCoSe}_{2}$ & -- & $1 \mathrm{~A} \mathrm{~g}^{-1}$ & $300.2 \mathrm{~F} \mathrm{~g}^{-1}$ & $2000,93.7 \%$ & 7 \\
\hline$(\mathrm{Ni}, \mathrm{Co}) \mathrm{Se}_{2} / \mathrm{NiCo}-\mathrm{LDH}$ & Special cactus-like structure & $2 \mathrm{~A} \mathrm{~g}^{-1}$ & $1224 \mathrm{~F} \mathrm{~g}^{-1}$ & $3000,75 \%$ & 8 \\
\hline $\mathrm{NiSe}_{2}$ & spheres & $1 \mathrm{~A} \mathrm{~g}^{-1}$ & $942.5 \mathrm{~F} \mathrm{~g}^{-1}$ & $1000,76 \%$ & 9 \\
\hline $\mathrm{NiCoSe}_{2}$ & $3 \mathrm{D}$ porous nanosheet & $1 \mathrm{~A} \mathrm{~g}^{-1}$ & $520 \mathrm{C} \mathrm{g}^{-1}$ & -- & 10 \\
\hline $\mathrm{MoSe}_{2}$ & Nanoflakes & $1 \mathrm{~A} \mathrm{~g}^{-1}$ & $435 \mathrm{~F} \mathrm{~g}^{-1}$ & $5000,92 \%$ & 11 \\
\hline $\mathrm{NiSe}_{2} @ \mathrm{CNT}$ & Nanosheets & $1 \mathrm{~A} \mathrm{~g}^{-1}$ & $980.5 \mathrm{~F} \mathrm{~g}^{-1}$ & $9000,82 \%$ & 12 \\
\hline $\mathrm{NiCo}_{2} \mathrm{Se}_{4} @$ carbon & litchi-like microspheres & $0.5 \mathrm{~A} \mathrm{~g}^{-1}$ & $1394 \mathrm{~F} \mathrm{~g}^{-1}$ & $\begin{array}{l}10000 \\
80.1 \%\end{array}$ & 13 \\
\hline $\mathrm{NiCo}_{2} \mathrm{Se}_{4} / \mathrm{RGO}$ & nanoneedles & $2 \mathrm{~A} \mathrm{~g}^{-1}$ & $1220 \mathrm{~F} \mathrm{~g}^{-1}$ & -- & 14 \\
\hline $\mathrm{NiCo}_{2} \mathrm{Se}_{4}$ & fluffy & $1 \mathrm{~A} \mathrm{~g}^{-1}$ & $602 \mathrm{C} \mathrm{g}^{-1}$ & $5000,96.8 \%$ & 15 \\
\hline $\mathrm{N}_{2} \mathrm{C}_{4} \mathrm{Se}$ & Hollow spheres & $\begin{array}{l}1 \mathrm{~A} \mathrm{~g}^{-1} \\
5 \mathrm{~A} \mathrm{~g}^{-1}\end{array}$ & $\begin{array}{l}1002 \mathrm{~F} \mathrm{~g}^{-1} \\
948 \mathrm{~F} \mathrm{~g}^{-1}\end{array}$ & $5000,80 \%$ & $\begin{array}{l}\text { This } \\
\text { work }\end{array}$ \\
\hline
\end{tabular}

\section{REFERENCES}

(1) Hou, L.; Shi, Y.; Wu, C.; Zhang, Y.; Ma, Y.; Sun, X.; Sun, J.; Zhang X.; Yuan,

C. Monodisperse Metallic NiCoSe 2 Hollow Sub-Microspheres: Formation Process, Intrinsic Charge - Storage Mechanism, and Appealing Pseudocapacitance as Highly Conductive Electrode for Electrochemical Supercapacitors. Adv. Funct. Mater. 2018, 28,1705921 
(2) Chang, A.; Zhang, C.; Yu, Y.; Yu Y.; Zhang, B. Plasma-Assisted Synthesis of $\mathrm{NiSe}_{2}$ Ultrathin Porous Nanosheets with Selenium Vacancies for Supercapacitor. ACS App. Mater. Interf. 2018, 10, 41861-41865.

(3) Lin, J.; Wang, H.; Yan, Y.; Zheng, X.; Jia, H.; Qi, J.; Cao, J.; Tu, J.; Fei W.; Feng, J. Core-branched $\mathrm{CoSe}_{2} / \mathrm{Ni}_{0.85} \mathrm{Se}$ nanotube arrays on $\mathrm{Ni}$ foam with remarkable electrochemical performance for hybrid supercapacitors. J. Mater. Chem. A 2018, 6, 19151-19158.

(4) Chen, T.; Li, S.; Wen, J.; Gui, P.; Guo, Y.; Guan, C.; Liu J.; Fang, G. Rational Construction of Hollow Core-Branch $\mathrm{CoSe}_{2}$ Nanoarrays for High-Performance Asymmetric Supercapacitor and Efficient Oxygen Evolution. Small 2018, 14, 1700979.

(5) Song, X.; Huang, C.; Qin, Y.; Li H.; Chen, H. C. Hierarchical hollow, sea-urchin-like and porous $\mathrm{Ni}_{0.5} \mathrm{Co}_{0.5} \mathrm{Se}_{2}$ as advanced battery material for hybrid supercapacitors. J. Mater. Chem. A 2018, 6, 16205-16212.

(6) Quan, L.; Liu, T.; Yi, M.; Chen, Q.; Cai D.; Zhan, H. Construction of hierarchical nickel cobalt selenide complex hollow spheres for pseudocapacitors with enhanced performance. Electrochim. Acta 2018, 281, 109-116.

(7) Miao, Y.; Sui, Y.; Zhang, D.; Qi, J.; Wei, F.; Meng, Q.; He, Y.; Sun Z.; Ren, Y. Polyhedral $\mathrm{NiCoSe}_{2}$ synthesized via selenization of metal-organic framework for supercapacitors. Mater. Lett. 2019, 242, 42-46.

(8) Li, X.; Wu, H.; Guan, C.; Elshahawy, A. M.; Dong, Y.; Pennycook S.J.; Wang, J. $(\mathrm{Ni}, \mathrm{Co}) \mathrm{Se}_{2} / \mathrm{NiCo}-\mathrm{LDH}$ Core/Shell Structural Electrode with the Cactus-Like S12 
(Ni,Co)Se $e_{2}$ Core for Asymmetric Supercapacitors. Small 2019, 15, 1803895.

(9) Yang, J.; Sun, Z.; Wang, J.; Zhang, J.; Qin, Y.; You J.; Xu, L. Hierarchical NiSe 2 spheres composed of tiny nanoparticles for high performance asymmetric supercapacitors. CrystEngComm 2019, 21, 994-1000.

(10)Wang, L.; Feng, Z.; Zhang, H.; Li, D.; Xing P. One-step electrodeposited 3D porous $\mathrm{NiCoSe}_{2}$ nanosheet array for high-performance asymmetric supercapacitors. Nanotechnology, 2020, 31,125403.

(11) Liu, Y.; Li, W.; Chang, X.; Chen, H.; Zheng, X.; Bai J.; Ren, Z. $\mathrm{MoSe}_{2}$ nanoflakes-decorated vertically aligned carbon nanotube film on nickel foam as a binder-free supercapacitor electrode with high rate capability.J. Colloid Interf. Sci. $2020,562,483-492$.

(12) Zheng, Y.; Tian, Y.; Sarwar, S.; Luo J.; Zhang, X. Carbon nanotubes decorated $\mathrm{NiSe}_{2}$ nanosheets for high-performance supercapacitors. J. Power Sources 2020, 452, 227793.

(13) Li, Y; Xu, L.; Jia, M.; Cui, L.; Gao, J.; Jin X. J. Hydrothermal Synthesis and Characterization of Litchi-Like $\mathrm{NiCo}_{2} \mathrm{Se}_{4} @$ carbon Microspheres for Asymmetric Supercapacitors with High Energy Density. J. Electrochem. Soc. 2018, 165 E303-E310.

(14) Ghosh, S.; Samanta, P.; Samanta, P.; Murmu, N. C.; Kuila, T. Investigation of Electrochemical Charge Storage Efficiency of $\mathrm{NiCo}_{2} \mathrm{Se}_{4} / \mathrm{RGO}$ Composites Derived at Varied Duration and Its Asymmetric Supercapacitor Device. Energy Fuel. 2020, 34, $13056-13066$. 
(15) Rajesh, J. A.; Lee, Y. H.; Yun, Y. H.; Quy, V. H.; Kang, S. H.; Kim, H.; Ahn, K. S. Bifunctional $\mathrm{NiCo}_{2} \mathrm{Se}_{4}$ and $\mathrm{CoNi}_{2} \mathrm{Se}_{4}$ nanostructures: Efficient electrodes for battery-type supercapacitors and electrocatalysts for the oxygen evolution reaction. J. Ind. Eng. Chem. 2019, 79, 370-382. 